\title{
Ocular Autoimmune Systemic Inflammatory Infectious Study (OASIS) —report 4: analysis and outcome of scleritis in an East Asian population
}

Muhammad Amir Bin Ismail ${ }^{1}$, Rachel Hui Fen Lim² ${ }^{2}$, Helen Mi Fang ${ }^{1}$, Elizabeth Poh Ying Wong ${ }^{1}$, Ho Su Ling ${ }^{1}$, Wee Kiak Lim ${ }^{1,3}$, Stephen C. Teoh ${ }^{1,3}$ and Rupesh Agrawal ${ }^{1 *}$ (D)

\begin{abstract}
Background: The purpose of this study is to evaluate the spectrum of scleritis from database of Ocular Autoimmune Systemic Inflammatory Infectious Study (OASIS) at a tertiary eye referral eye institute in Singapore. Clinical records of 120 patients with scleritis from a database of 2200 patients from Ocular Autoimmune Systemic Inflammatory Infectious Study (OASIS) were reviewed.

Results: $56.6 \%$ were females, with a mean age of $48.6 \pm 15.9$ years. 75 (62.5\%) had diffuse anterior scleritis, 25 (20. $8 \%)$ had nodular anterior scleritis, 7 (5.8\%) had necrotizing anterior scleritis and 13 (10.8\%) had posterior scleritis. Ocular complications were observed in $53.3 \%$ of patients, including anterior uveitis (42.5\%), raised intraocular pressure (12.5\%), and corneal involvement (11.7\%). Autoimmune causes were associated with 31 (25.8\%) of patients, and 10 (8.3\%) patients had an associated infective etiology, much higher than Caucasian studies. 53.3\% of patients were treated with oral corticosteroids while $26.7 \%$ required immunosuppressives.

Conclusions: Infective etiology needs to be considered in patients of scleritis from Asian origin. In our study and in OASIS database, scleritis was associated with systemic autoimmune disease and ocular complications.
\end{abstract}

Keywords: Scleritis, Epidemiology, Complications, Treatment

\section{Background}

Scleritis is an uncommon potentially sight threatening ocular inflammatory disease $[1,2]$. It is typically a severe painful inflammatory process characterized by inflammation of the sclera associated with engorgement of the conjunctival, superficial, and deep episcleral vessels. Based on the Watson and Hayreh classification system [3], scleritis is divided into anterior and posterior types. The anterior type is further subdivided into diffuse, nodular, and necrotizing with or without inflammation.

Ocular complications include scleral and corneal thinning, perforation, keratitis, anterior uveitis, glaucoma, cataract, and exudative retinal detachment $[1,3]$.

\footnotetext{
* Correspondence: Rupesh_agrawal@ttsh.com.sg

${ }^{1}$ National Healthcare Group Eye Institute, Tan Tock Seng Hospital, Singapore 308433, Singapore

Full list of author information is available at the end of the article
}

Necrotizing scleritis, in particular, has been more frequently associated with ocular complications $[3,4]$.

The disease may be idiopathic but previous studies have suggested that scleritis is often associated with, and may be the first sign of potentially life-threatening systemic autoimmune diseases in $30-50 \%$. The most common are rheumatoid arthritis, granulomatosis with polyangiitis (formerly Wegener's granulomatosis), seronegative spondyloarthropathies, relapsing polychondritis, and systemic lupus erythematosus (SLE) [5-10]. It may also be associated with infectious causes, previous ocular surgery, drugs, or malignancies [2, 6, 7].

Management of scleritis involves a multidisciplinary approach when associated with a systemic disease. Treatment is in a stepwise manner with non-steroidal anti-inflammatory drugs (NSAIDs), followed by corticosteroids, and subsequently, steroid sparing immunosuppressive 
agents in cases of unsatisfactory therapeutic response or if prolonged treatment is necessary. Examples of such agents are azathioprine, cyclosporine, methotrexate, cyclophosphamide, and mycophenolate mofetil (MMF) [11-13]. In cases of refractory or therapy-resistant ocular inflammatory eye disease, tumor necrosis factor alpha (TNF- $\alpha$ ) antagonists such as infliximab and adalimumab are becoming increasingly used, together with other immunosuppressive agents [14-20]. More recently, rituximab, a CD20 monoclonal antibody, has also been described for refractory scleritis [21].

As scleritis is a relatively uncommon disorder, there are limited large studies documenting clinical experience with this disease, especially in Asian populations [2224]. We aim to analyze our clinical experience with this disorder, in particular, the associated systemic diseases, complications, treatments methods, and outcomes of patients with scleritis presenting to a tertiary institution in Singapore from a very large database of ocular inflammatory disease-Ocular Autoimmune Systemic Inflammatory Infectious Study (OASIS) database.

\section{Results}

One hundred and twenty patients (5.45\%) with scleritis were identified during this 11-year period, out of a total of 2200 patients that presented to our Uveitis and Ocular Inflammation service. The detailed demographic and clinical characteristics of all patients included in this study are summarized in Table 1 . There were 52 (43.3\%) males and 68 (56.7\%) females. The mean age was $48.6 \pm$ 15.9 (range 16-83) years. Eighty three patients (69.2\%) were Chinese, 15 (12.5\%) Indian, 14 (11.7\%) Malay, and $8(6.7 \%)$ patients of other races. The disease was bilateral in 37 patients $(30.8 \%)$. The commonest presenting complaints included eye redness $(n=105,87.5 \%)$, ocular pain $(n=80,66.7 \%)$, and blurring of vision $(n=21,17.5 \%)$.

Diffuse anterior scleritis $(n=75,62.5 \%)$ was the most common type of scleritis, followed by nodular $(n=25$, $20.8 \%)$, posterior $(n=13,10.8 \%)$ and necrotizing anterior scleritis $(n=7,5.8 \%)$. Demographic and clinical characteristics of the subtypes of scleritis are summarized in Table 2. The mean age at presentation was highest for necrotizing anterior scleritis (63.7 \pm 16.5 years) compared to other forms of scleritis $(48.6 \pm 14.1$ years for diffuse, $44.0 \pm 16.9$ for nodular, and $49.2 \pm 20.3$ for posterior). There were statistically significant differences in age among the 4 subtypes of scleritis $(p=0.037)$. Post hoc pairwise comparisons showed that patients in total necrotizing subtype were 19.7 years older than patients in nodular subtype (95\% confidence interval (CI) from 1.8 to 37.5 , adjusted $p=0.022$ ).

\section{Complications}

At least one ocular complication was observed in 53.3\% of all patients either at presentation or at follow up.
Corneal involvement (ulceration, keratitis), high intraocular pressure, and anterior uveitis constituted major anterior segment complications while vitritis, cystoid macular edema, exudative retinal detachment, and optic disc swelling were the major posterior segment complications. Ocular complications were present in $49.3 \%$ ( $n$ $=37)$ of diffuse, $40.0 \%(n=10)$ of nodular, $85.7 \%(n=6)$ of necrotizing, and $84.6 \%(n=11)$ of posterior scleritis. Posterior scleritis and necrotizing scleritis were associated with higher rates of ocular complications $(p=$ 0.014). The most common complication was anterior uveitis, which occurred in 51 (42.5\%) of patients.

Posterior segment complications were most commonly observed in patients with posterior scleritis $(p<0.001)$. This included exudative retinal detachment (RD) (30.8\%, $n=4)$, cystoid macular edema $(23.1 \%, n=3)$, and optic disc swelling $(15.4 \%, n=2)$.

\section{Systemic associations}

Associated autoimmune and infective associations are summarized in Table 3. Autoimmune and infective causes were associated with $31(25.8 \%)$ and $10(8.3 \%)$ of scleritis patients, respectively. The most common associated autoimmune cause was rheumatoid arthritis (7.5\%), followed by granulomatosis with polyangiitis $(4.2 \%)$, relapsing polychondritis (3.3\%), Sjogren's syndrome (3.3\%), and psoriatic arthropathy (1.7\%). Necrotizing anterior scleritis had the highest proportion with an autoimmune condition (85.7\%), followed by posterior scleritis (30.8\%), and diffuse scleritis (25.3\%), $p=0.001$. Only 2 out of $25(8.0 \%)$ patients with nodular scleritis patients had an associated autoimmune conditions.

Infective screen was done in 41 patients. The most commonly associated infective cause was positive test for latent mycobacterial infection. Ten showed a positive TB T spot, and 3 were indeterminate. A disproportionately high percentage of nodular scleritis (6 patients out of 25 , or $24 \%$ ) were associated with a possible infective cause, while only $8 \%$ had an associated autoimmune cause. Nodular scleritis was found to have a higher association with a probable infective etiology.

\section{Treatment}

Treatment modalities for all patients are shown in Table 4. One hundred and fourteen (95\%) were treated with topical corticosteroids as first-line treatment. Sixtythree patients $(52.5 \%)$ were treated oral NSAIDs, 64 (53.3\%) patients were treated with oral corticosteroids, and $26.7 \%$ needed immunosuppressants including azathioprine $(n=16,13.3 \%)$, methotrexate $(n=11,9.2 \%)$, MMF $(n=6,5 \%)$, sulphasalazine $(n=6,5 \%)$, hydroxychloroquine $(n=5,4.2 \%)$, cyclophosphamide $(n=4$, $3.3 \%)$, and cyclosporine $(n=4,3.3 \%)$. Intravenous methylprednisolone was given to 5 patients $(4.2 \%)$, and 2 
Table 1 Demographic and clinical characteristics of patients with scleritis from Ocular Autoimmune Systemic Inflammatory Infectious Study (OASIS) database

\begin{tabular}{|c|c|}
\hline Characteristics & No. of patients, $n(\%)$ \\
\hline Mean age $\pm S D$ (years) & $48.6 \pm 15.9$ \\
\hline Range & $16-83$ \\
\hline \multicolumn{2}{|l|}{ Gender } \\
\hline Male & $52(43.3 \%)$ \\
\hline Female & $68(56.7 \%)$ \\
\hline Bilaterality & $37(30.8 \%)$ \\
\hline \multicolumn{2}{|l|}{ Race } \\
\hline Chinese & $83(69.2 \%)$ \\
\hline Indian & $15(11.7 \%)$ \\
\hline Malay & $14(11.7 \%)$ \\
\hline Others & $8(6.7 \%)$ \\
\hline Ocular complications (any) & $64(53.3 \%)$ \\
\hline \multicolumn{2}{|l|}{ Anterior segment } \\
\hline Anterior uveitis & $51(42.5 \%)$ \\
\hline Corneal involvement (ulceration, keratitis) & $14(11.7 \%)$ \\
\hline Ocular hypertension & $15(12.5 \%)$ \\
\hline \multicolumn{2}{|l|}{ Posterior segment } \\
\hline Cystoid macular edema & $3(2.5 \%)$ \\
\hline Exudative retinal detachment & $5(4.2 \%)$ \\
\hline Optic disc swelling & $3(2.5 \%)$ \\
\hline \multicolumn{2}{|l|}{ Systemic associations } \\
\hline Autoimmune & $31(25.8 \%)$ \\
\hline Rheumatoid arthritis & $9(7.5 \%)$ \\
\hline Granulomatosis with polyangitis & $5(4.2 \%)$ \\
\hline Relapsing polychondritis & $4(3.3 \%)$ \\
\hline Sjogren's syndrome & $4(3.3 \%)$ \\
\hline Psoriatic arthropathy & $2(1.7 \%)$ \\
\hline Systemic lupus erythematosus & $1(0.8 \%)$ \\
\hline Behcet's disease & $1(0.8 \%)$ \\
\hline Churg-Strauss syndrome & $1(0.8 \%)$ \\
\hline Ulcerative colitis & $1(0.8 \%)$ \\
\hline Takayasu's arteritis & $1(0.8 \%)$ \\
\hline Non-specific autoimmune disease & $1(0.8 \%)$ \\
\hline Orbital inflammatory syndrome & $1(0.8 \%)$ \\
\hline Other non-autoimmune systemic: & $2(1.7 \%)$ \\
\hline Myelodysplastic syndrome & $1(0.8 \%)$ \\
\hline Polyclonal gammopathy & $1(0.8 \%)$ \\
\hline Presumed infectious association & $10(8.3 \%)$ \\
\hline TB T-spot positive & $5(4.2 \%)$ \\
\hline Herpes zoster ophthalmicus & $2(1.7 \%)$ \\
\hline Herpes simplex viral keratitis & $1(0.8 \%)$ \\
\hline Acquired immunodeficiency syndrome & $1(0.8 \%)$ \\
\hline
\end{tabular}

Table 1 Demographic and clinical characteristics of patients with scleritis from Ocular Autoimmune Systemic Inflammatory Infectious Study (OASIS) database (Continued)

\begin{tabular}{ll}
\hline Hansen's disease & $1(0.8 \%)$ \\
Recurrence & $20(16.7 \%)$ \\
Treatment & \\
Topical steroids & $114(95.0 \%)$ \\
Oral non-steroidal anti-inflammatory drugs & $63(47.3 \%)$ \\
Oral corticosteroids & $64(53.3 \%)$ \\
Systemic immunosuppressant (any) & $32(26.7 \%)$ \\
Azathioprine & $16(13.3 \%)$ \\
Methotrexate & $11(9.2 \%)$ \\
Mycophenolate mofetil & $6(5.0 \%)$ \\
Sulphasalazine & $6(5.0 \%)$ \\
Cyclosporine & $4(3.3 \%)$ \\
Cyclophosphamide & $4(3.3 \%)$ \\
Tumor necrosis factor-a antagonist (infliximab) & $2(1.7 \%)$ \\
\hline
\end{tabular}

patients required biologic agents (intravenous Infliximab) (1.7\%). The choices of oral NSAIDs or systemic steroids/immunosuppressants were made clinically, depending on the severity of the scleritis.

Among the 100 patients with non-necrotizing (diffuse and nodular) anterior scleritis, $56(56.0 \%)$ received oral NSAIDs as first-line therapy, with 96 patients (96.0\%) treated with topical steroids. Out of the 56 patients receiving NSAID therapy, $41(41.0 \%)$ responded and required no further treatment. Fifteen patients (15.0\%) were unresponsive to therapy and were given either systemic corticosteroids alone or in combination with immunosuppressive drugs. For 31 patients (31.0\%), the first choice treatment was systemic corticosteroids.

Oral corticosteroids were used in $85.7 \%$ of necrotizing anterior scleritis and $92.3 \%$ of posterior scleritis, compared to $49.3 \%$ of diffuse scleritis and $32 \%$ of nodular scleritis. Around $70 \%$ of necrotizing anterior scleritis and $38.5 \%$ of posterior scleritis patients required immunosuppressive agents while only $24.0 \%$ and $12.0 \%$ of diffuse and nodular scleritis required immunosuppressive treatment.

Anti-tubercular therapy for 6 months was given in the patients with latent tubercular disease.

\section{Recurrence and resolution}

Recurrence of scleritis occurred in 20 patients (16.7\%), with the largest proportion of recurrence amongst patients with necrotizing anterior scleritis $(n=2,26.6 \%)$, followed by diffuse anterior scleritis $(n=14,18.7 \%)(p=0.463)$.

Resolution of inflammation was longer amongst patients with necrotizing sclerosis, and occurred in 
Table 2 Demographic and clinical characteristics of patients classified into subtypes of scleritis (no. of patients, n (\%))

\begin{tabular}{|c|c|c|c|c|}
\hline & Diffuse & Nodular & Necrotizing & Posterior \\
\hline Number of patients & $75(62.5)$ & $25(20.8)$ & $7(5.8)$ & $13(10.8)$ \\
\hline Age (mean) years & 48.6 & 44.0 & 63.7 & 49.2 \\
\hline Range & $20-74$ & $18-79$ & $38-83$ & $16-80$ \\
\hline \multicolumn{5}{|l|}{ Gender } \\
\hline Male & $32(42.7)$ & $12(48.0)$ & $2(28.6)$ & $5(38.5)$ \\
\hline Female & $43(57.3)$ & $13(52.0)$ & $5(71.4)$ & $8(61.5)$ \\
\hline Ocular complications (any) & $37(49.3)$ & $10(40.0)$ & $6(85.7)$ & $12(84.6)$ \\
\hline \multicolumn{5}{|l|}{ Anterior segment } \\
\hline Corneal involvement (ulceration, keratitis) & $7(9.3)$ & $4(16.0)$ & $2(28.6)$ & $1(7.7)$ \\
\hline Ocular hypertension & $9(12.0)$ & $2(8.0)$ & $2(28.6)$ & $2(15.4)$ \\
\hline Anterior uveitis & $30(40.0)$ & $8(32.0)$ & $5(71.4)$ & $8(61.5)$ \\
\hline \multicolumn{5}{|l|}{ Posterior segment } \\
\hline Vitritis & 0 & 0 & 0 & $3(23.1)$ \\
\hline Cystoid macular edema & 0 & 0 & 0 & $3(23.1)$ \\
\hline Exudative retinal detachment & 0 & 0 & $1(14.3)$ & $4(30.8)$ \\
\hline Optic disc swelling & $1(1.3)$ & 0 & 0 & $2(15.4)$ \\
\hline
\end{tabular}

19.3 days in diffuse, 21.2 days in nodular. and 75 days in necrotizing scleritis.

\section{Discussion}

Scleritis is an uncommon inflammatory disease that may cause complications if not treated in a timely manner. This retrospective study involved 120 patients with scleritis seen over an 11-year period in a tertiary eye center in Singapore.

The mean age of our patients were 48.6, slightly younger compared to the Caucasian population (51.5 years) [9]. Our female to male ratio was $1.3: 1$, similar to the rates reported in the literature $(1.27: 1$ and $1.57: 1)$ [4, 9, 27]. Around $31 \%$ of patients had bilateral disease, similar to the data reported by Sainz de la Maza et al. (34\%) [4].

Laboratory evaluation revealed positive autoimmune markers in 34 patients $(28.3 \%)$. Fourteen (11.7\%) were RF

Table 3 Associated autoimmune and systemic disease (no. of patients, $n(\%))$

\begin{tabular}{lllll}
\hline & Diffuse & Nodular & Necrotizing & Posterior \\
\hline Number of patients & $75(62.5)$ & $25(20.8)$ & $7(5.8)$ & $13(10.8)$ \\
$\begin{array}{l}\text { Systemic autoimmune } \\
\text { disease }\end{array}$ & $19(25.3)$ & $2(8.0)$ & $6(85.7)$ & $4(30.8)$ \\
$\begin{array}{l}\text { Rheumatoid arthritis } \\
\text { Granulomatosis with }\end{array}$ & $6(5.0)$ & 0 & $3(42.9)$ & 0 \\
polyangitis & & 0 & 0 & $2(15.4)$ \\
$\begin{array}{l}\text { Relapsing polychondritis } \\
\text { Psoriatic arthropathy }\end{array}$ & $2(2.7)$ & $1(4.0)$ & $1(14.3)$ & 0 \\
$\begin{array}{l}\text { Presumed infectious } \\
\text { association }\end{array}$ & $4(5.3)$ & $6(24.0)$ & $0(0)$ & 0 \\
\hline
\end{tabular}

positive, 13 (10.8\%) were ANA positive, 9 (7.5\%) were ANCA positive, and 3 (2.5\%) were anti-dsDNA (DsDNA) positive. Out of these, three were newly diagnosed with RA, three granulomatosis with polyangiitis, and one with SLE. Our results showed that laboratory investigations are useful in diagnosing an underlying systemic disease associated with scleritis. Screening for an underlying systemic disease should be aimed at diseases with higher associations such as RA and granulomatosis with polyangiitis. As presence of scleritis is associated with a worse prognosis and a higher mortality rate in patients with rheumatoid arthritis, initial systemic evaluation and close follow-up of patients with idiopathic scleritis and rheumatoid factor positive is crucial [25].

Previous series from North America and Europe have demonstrated that systemic autoimmune diseases are present in approximately $30-50 \%$ of patients with scleritis [3, 4, 26] In our study, $25.8 \%$ had a systemic rheumatologic disease. This is similar to other Asian studies which showed an association in $15-22 \%$ of patients

Table 4 Treatment and recurrence (no. of patients, $n(\%)$ )

\begin{tabular}{lllll}
\hline & Diffuse & Nodular & Necrotizing & Posterior \\
\hline Number of patients & $75(62.5)$ & $25(20.8)$ & $7(5.8)$ & $13(10.8)$ \\
Topical steroids & $71(94.7)$ & $25(100)$ & $6(85.7)$ & $12(92.3)$ \\
Oral NSAID & $40(53.3)$ & $16(64.0)$ & $3(42.9)$ & $4(30.8)$ \\
Oral corticosteroids & $37(49.3)$ & $9(36.0)$ & $6(85.7)$ & $12(92.3)$ \\
$\begin{array}{l}\text { Oral immunosuppressives } \\
\text { (excluding prednisolone) }\end{array}$ & $19(25.3)$ & $3(12.0)$ & $5(71.4)$ & $5(38.5)$ \\
$\begin{array}{l}\text { Oral steroid or } \\
\text { immunosuppressives }\end{array}$ & $40(53.3)$ & $9(36.0)$ & $6(85.7)$ & $12(92.3)$ \\
Recurrence & $14(18.7)$ & $2(8.0)$ & $2(28.6)$ & $2(15.4)$ \\
\hline
\end{tabular}


[24, 27-29]. Our findings similarly suggest that the association with systemic diseases might be lower in Asian populations with scleritis compared to North American and European populations [30, 31]. The most common rheumatologic disease is RA, followed by granulomatosis with polyangiitis, relapsing polychondritis, Sjogren's syndrome, and psoriatic arthropathy. A comparison of the results from our study with previously published data (other Asian, European, and North American studies) are summarized in Table 5.

Associated infective screen was positive in 10 patients (8.3\%), with TB being the most common in our study. Three of the patients with presumed TB-related nodular scleritis were foreign nationals working in Singapore ( 2 from China, and 1 from India).

Based on current literature, an infectious cause is found in $4-18 \%$ of patients, of which herpes zoster is the most common, followed by TB, syphilis, leprosy, and Lyme borreliosis [3, 6, 9, 32]. A study by Tabara [33] has suggested that TB might be involved in the pathogenesis of scleritis in some patients, particularly in eyes that present with localized elevated nodules of the sclera. Asian patients with nodular scleritis may benefit from screening for an infective agent such as TB, as they will require a different therapeutic approach. It is also important to note that TB is still an important infective cause in the Asian patient who presents with scleritis.

In addition to autoimmune diseases and infections, it is also important to bear in mind that a malignancy can be the underlying disease in a patient with scleritis. Posterior scleritis has been reported in patients with lymphoma, multiple myeloma, and Waldenstrom macroglobulinemia [34]. In our study, there was one patient with myelodysplastic syndrome and another with polyclonal gammopathy. Medication, surgery, and trauma are reported to be rare in all studies.

Management of non-necrotizing anterior scleritis consists of NSAIDs in combination with topical corticosteroids as first line based on published data [2, 5, 35, 36]. $21 \%$ of patients with non-necrotizing anterior scleritis

Table 5 Comparison of the results from our series with previously published data (\%)

\begin{tabular}{lclll}
\hline & $\begin{array}{l}\text { Our } \\
\text { study }\end{array}$ & $\begin{array}{l}\text { Other Asian } \\
\text { Studies } \\
{[24,29-31]}\end{array}$ & $\begin{array}{l}\text { European } \\
\text { studies } \\
{[32,33]}\end{array}$ & $\begin{array}{l}\text { North } \\
\text { American } \\
\text { studies [27] }\end{array}$ \\
\hline $\begin{array}{l}\text { Associated } \\
\text { autoimmune } \\
\text { disease }\end{array}$ & 25.8 & $15-22$ & $32.7-52$ & $35.8-39$ \\
$\begin{array}{l}\text { Infectious } \\
\text { cause (TB) }\end{array}$ & 4.2 & 7.2 & 0 & $0-2$ \\
$\begin{array}{l}\text { Systemic steroid } \\
\text { therapy }\end{array}$ & 53.3 & 42.2 & 61.5 & $31.9-36.7$ \\
$\begin{array}{l}\text { Immunosuppressive } \\
\text { therapy }\end{array}$ & 26.7 & 22.9 & $34-36$ & $26.1-38.0$ \\
\hline
\end{tabular}

required additional immunosuppressive agents while $85.7 \%$ necrotizing, and $30.8 \%$ of posterior scleritis required immunosuppressive therapy. In the study by Jabs et al. [8], nearly $67 \%$ of patients with scleritis required more than NSAID therapy, and 26\% needed immunosuppressant therapy. In our study, we found a similar treatment pattern with $65.8 \%$ of patients needing more than NSAIDs to control the inflammation, and 25.8\% were treated with immunosuppressive drugs.

TNF- $\alpha$ (infliximab) is a biologic immunomodulatory agent used as first line for Behcet's disease or for ocular complications of rheumatoid arthritis when patients have failed combination treatment with one or more immunosuppressive agents [35] In our series, 2 patients (1.7\%) were started on infliximab treatment. One was due to necrotizing anterior scleritis secondary to relapsing polychondritis, and another due to nodular scleritis secondary to rheumatoid arthritis. Both failed treatment with corticosteroids, azathioprine, cyclosporine, and mycophenolate mofetil. In general, for this study, intravenous infliximab were used only after failure of several different types of immunosuppressive agents in a step ladder increment, due to financial factors as insurance does not cover the costs of these new agents locally.

Resolution of inflammation occurred in 19.3 days in diffuse, 21.2 days in nodular, and 75 days in necrotizing scleritis. In the study by Tuft and Watson [9], patients with diffuse scleritis received treatment for the shortest period, whereas the mean duration of treatment was longest for the necrotizing group. This finding is consistent with our study which showed that resolution of inflammation is shortest in diffuse and longest in necrotizing scleritis. Recurrence rate is highest among the necrotizing scleritis, with $28.6 \%$ of patients having at least 1 recurrence.

The limitations of our study lie mainly in the retrospective nature, with a relatively small sample size collected from a single institution. This may not fully reflect the spectrum of scleritis of our entire local population. Furthermore, the treatment modalities may differ depending on the preferences of the ocular inflammation specialists. Nonetheless, this is a relatively uncommon condition, and our data was obtained over a long 11-year period. The results of our study add to the knowledge of the epidemiology of scleritis in an Asian clinical setting.

\section{Conclusions}

In conclusion, scleritis is a severe ocular inflammation which may be idiopathic, or associated with autoimmune or infectious cause. While infectious causes are uncommon, Asian patients with nodular scleritis may need screening for an infective agent such as $\mathrm{TB}$ as this may alter the management of this disease. Treatment may involve a step ladder management strategy with oral 
NSAIDs, oral corticosteroids, and immunosuppressants/ biologic agents. Close monitoring of complications, especially for necrotizing scleritis and posterior scleritis, systemic evaluation, and timely treatment are necessary for these patients.

\section{Methods}

We retrospectively analyzed the case records of all consecutive new scleritis cases diagnosed at the Uveitis and Ocular Inflammation service of our hospital, from January 2004 to December 2014 from 2200 patients in the OASIS database.

Data collected included information such as age at diagnosis, race, gender, anatomic location of inflammation, presenting complaints, ocular complications, associated systemic or inflammatory disease, investigations, use of topical and/or systemic treatments, and recurrence.

The diagnosis of scleritis was based on a comprehensive clinical history including a systemic review, detailed ocular examination, and investigations when deemed necessary. The Watson system was used to classify the type of scleritis [3], with the diagnosis of posterior scleritis confirmed by ultrasonography and/or CT of the orbit [34]. In all the cases, the diagnosis of scleritis was made by a uveitis specialist in our ocular inflammation service.

Complications were divided into anterior and posterior segment complications. Corneal complications included ulcerative or peripheral thinning, while anterior uveitis was diagnosed when $1+$ cells (based on the Standardization of Uveitis Nomenclature Working Group Criteria) [37] or more were observed in the anterior chamber. Ocular hypertension was defined as an intraocular pressure of more than $21 \mathrm{mmHg}$ measured by the Goldmann applanation tonometer (GAT). The presence of cystoid macular edema was noted clinically and confirmed by further investigations such as optical coherence tomography or fundus fluorescein angiography. Serous retinal detachment was diagnosed on fundoscopy or ultrasound.

Laboratory testing included blood tests, urine tests, and a chest radiograph. Blood tests included a complete blood count $(\mathrm{CBC})$ and white cell differential, acute phase reactants such as erythrocyte sedimentation rate (ESR) and C-reactive protein (CRP), liver and renal function tests. Autoimmune markers such as rheumatoid factor (RF), anti-nuclear antibodies (ANA), anti-doublestranded DNA (anti-dsDNA), anti-neutrophil cytoplasmic antibody (ANCA) were also included. Other tests, which include infective screening for tuberculosis (TB) using the tuberculosis interferon gamma release assay (T-SPOT.TB, Oxford Immunotec, UK), treponemal serology (syphilis IgG and Venereal Disease Research Laboratory (VDRL)), were not taken routinely, but when deemed necessary based on history and physical examination. A history of scleritis with discharge, or associated trauma, and examination findings of a yellowish nodular lesion was deemed more suspicious for an infective cause and further investigations were performed.

Associated systemic diseases were classified into infectious or autoimmune. These were diagnosed based on the results of a compatible history, clinical features, and laboratory data, often in conjunction with rheumatologists and infectious disease physicians.

Treatment involved oral NSAIDs, topical, and/or systemic corticosteroids depending on the severity, location, and chronicity of the inflammatory process, as well as the risk of visual loss and complications. Immunosuppressive drugs were used as a second-line therapy when there was failure of response to oral corticosteroid monotherapy, development of intolerable side effects to corticosteroids, or in the presence of associated systemic rheumatological disease.

Recurrence was defined as repeated episodes of scleritis separated by periods of inactivity over 3 months in duration without treatment. The duration of follow-up was also obtained.

All data were entered into a computerized database system for descriptive analysis and analyzed with IBM SPSS Statistics software (version 22, IBM, New York, USA) and $\mathrm{R}$ (version 3.1.2, The $\mathrm{R}$ Foundation for Statistical Computing), with continuous variables expressed as mean with standard deviation, and range and categorical variables as percentages. Pearson's chisquare test was performed to analyze association between the groups of patients and their demographics and clinical characteristics. If expected counts within a category were less than 5 for more than $20 \%$ of cells, Fisher's exact test was used instead. Age among the groups of patients were compared using one way analysis of variance (ANOVA), and post hoc pairwise comparisons done with Bonferroni correction applied if the ANOVA showed results of statistical significance. A $p$ value of $<0.05$ was considered to indicate statistical significance.

The study was approved by the National Healthcare Group Domain Specific Review Board, the conduct of which is overseen by the National Healthcare Group Research Ethics Committee.

\section{Acknowledgements \\ NIL.}

\section{Authors' contributions}

RA is the overall consultant overseeing and editing the paper. MABI is the first author of the paper and is responsible for the first draft of the paper and data collection. RLHF participated in the data collection. HMF is the second author of the paper and is responsible for data analysis and editing the manuscript. EPYW is the statistician and participated in the data analysis. HSL, LWK, and ST are the consultants overseeing and editing the paper. All authors read and approved the final manuscript. 


\section{Competing interests}

The authors report no conflicts of interest. The authors alone are responsible for the content and writing of the paper. The intent of this policy is not to prevent authors with these relationships from publishing work, but rather to adopt transparency such that readers can make objective judgments on conclusions drawn.

\section{Author details}

'National Healthcare Group Eye Institute, Tan Tock Seng Hospital, Singapore 308433, Singapore. ${ }^{2}$ Singapore National Eye Centre, Singapore, Singapore.

${ }^{3}$ Eagle Eye Center, Mount Elizabeth Novena Hospital, Singapore, Singapore.

Received: 27 October 2016 Accepted: 2 February 2017

Published online: 15 February 2017

\section{References}

1. McCluskey PJ, Wakefield D (1996) Scleritis and Episcleritis. In: Pepose JS, Holland GN, Wilhelms KR (eds) Ocular inflammation and immunity. Mosby, St Louis, pp 642-647

2. Okhravi N, Odufuwa B, McCluskey P et al (2005) Scleritis. Surv Ophthalmol 50:351-363

3. Watson PG, Hayreh SS (1976) Scleritis and Episcleritis. Br J Ophthalmol 60: 163-191

4. Sainz de la Maza M, Jabbur NS, Foster CS (1994) Severity of scleritis and episcleritis. Ophthalmology 101:389-396

5. Albini TA, Rao NA, Smith RE (2005) The diagnosis and management of anterior scleritis. Int Ophthalmol Clin 45:191-204

6. Smith JR, Mackensen F, Rosenbaum JT (2007) Therapy insight: scleritis and its relationship to systemic autoimmune disease. Nat Clin Pract Rheumato 3:219-226

7. Pavesio CE, Meier FM (2001) Systemic disorders associated with episcleritis and scleritis. Curr Opin Ophthalmol 12:471-478

8. Jabs DA, Mudun A, Dunn JP et al (2000) Episcleritis and scleritis: clinical features and treatment results. Am J Ophthalmol 130:469-476

9. Tuft SJ, Watson PG (1991) Progression of scleral disease. Ophthalmology 98: 467-471

10. Lin P, Bhullar SS, Tessler HH et al (2008) Immunologic markers as potential predictors of systemic autoimmune disease in patients with idiopathic scleritis. Am J Ophthalmol 145:463-471

11. Wakefield D, McCluskey P (1989) Cyclosporin therapy for severe scleritis. Br J Ophthalmol 73:743-746

12. Shah SS, Lowder CY, Schmitt MA et al (1992) Low dose methotrexate therapy for ocular inflammatory disease. Ophthalmology 99:1419-1423

13. Throne EJ, Jabs DA, Qaiz FA et al (2005) Mycophenolate mofetil therapy for inflammatory eye disease. Ophthalmology 112:1472-1477

14. Murphy CC, Ayliffe WH, Booth A et al (2004) Tumor necrosis factor alpha blockade with infliximab for refractory uveitis and scleritis. Ophthalmology 111:352-356

15. Chauhan S, Kamal A, Thompson RN et al (2009) Rituximab for treatment of scleritis associated with rheumatoid arthritis. Br J Ophthalmol 93:984-985

16. Doctor P, Sultan A, Syed $S$ et al (2010) Infliximab for the treatment of refractory scleritis. Br J Ophthalmol 94:579-583

17. laccheri B, Androudi S, Bocci EB et al (2010) Rituximab treatment for persistent scleritis associated with rheumatoid arthritis. Ocul Immunol Inflamm 18:223-225

18. Kurz PA, Suhler EB, Choi D, Rosenbaum JT (2009) Rituximab for treatment of ocular inflammatory disease: a series of four cases. Br J Ophthalmol 93:546-548

19. Restrepo JP, Molina MP (2010) Successful treatment of severe nodular scleritis with adalimumab. Clin Rheumatol 29:559-561

20. Sen HN, Sangave A, Hammel K et al (2009) Infliximab for the treatment of active scleritis. Can J Ophthalmol 44:e9-e12

21. Suhler EB, Lim LL, Beardsley RM et al (2014) Rituximab therapy for refractory scleritis: results of a phase I/II dose-ranging, randomized, clinical trial. Ophthalmology 121(10):1885-1891

22. Nakajima N, Hayasaka S (1995) Clinical features of episcleritis and scleritis in some Japanese patients. Ophthalmologica 209:256e9

23. Anshu A, Chee SP (2007) Posterior scleritis and its association with HLA B27 haplotype. Ophthalmologica 221:275e8

24. Keino H, Watanabe T, Taki W et al (2010) Clinical features and visual outcomes of Japanese patients with scleritis. Br J Ophthalmol 94:1459-1463
25. Sainz de la Maza M, Foster CS, Jabbur NS (1995) Scleritis associated with systemic vasculitic diseases. Ophthalmology 102:687-692

26. McGavin DDM, Williamson J, Forrester JV et al (1976) Episcleritis and scleritis. A study of their clinical manifestation and association with rheumatoid arthritis. Br J Ophthalmol 60:192e226

27. Araki K, Nakagawa Y, Tada R et al (1987) Clinical features of 75 cases of scleritis during the past 11 years. Jpn J Clin Ophthalmol 41:1075e8

28. Kurosaka H, Muraki Y, Suzuki S (1994) Evaluation of 106 cases of scleritis in our hospital. Folia Ophthalmol Jpn 45:797e803

29. Zhou Y, Wang Z, Zhang $Y$ et al (2014) Study on the clinical manifestation of 90 cases with scleritis and episcleritis. Zhonghua Yan Ke Za Zhi 50(4):261-266

30. Berchicci L, Miserocchi E, Di Nicola M et al (2014) Clinical features of patients with episcleritis and scleritis in an Italian tertiary care referral center. Eur J Ophthalmol 24(3):293-298

31. Wieringa WG, Wieringa JE, ten Dam-van Loon NH et al (2013) Visual outcome, treatment results, and prognostic factors in patients with scleritis. Ophthalmology 120(2):379-386

32. Cobo M (1983) Inflammation of the sclera. Int Ophthalmol Clin 23:159-171

33. Tabbara KF (2007) Tuberculosis. Curr Opin Ophthalmol 18:493e501

34. McCluskey PJ, Watson PG, Lightman S, Scleritis P et al (1999) Clinical features, systemic associations, and outcome in a large series of patients. Ophthalmology 106:2380-2386

35. Kim EC, Foster CS (2006) Immunomodulatory therapy for the treatment of ocular inflammatory disease: evidence-based medicine recommendations for use. Int Ophthalmol Clin 46:141-164

36. Sainz de la Maza M, Jabbur NS, Foster CS (1993) An analysis of therapeutic decision for scleritis. Ophthalmology 100:1372-1376

37. Jabs DA, Nussenblatt RB, Rosenbaum JT (2005) Standardization of Uveitis Nomenclature (SUN) Working Group. Standardization of uveitis nomenclature for reporting clinical data. Results of the First International Workshop. Am J Ophthalmol 140(3):509-516

\section{Submit your manuscript to a SpringerOpen ${ }^{\circ}$ journal and benefit from:}

- Convenient online submission

- Rigorous peer review

- Immediate publication on acceptance

- Open access: articles freely available online

- High visibility within the field

- Retaining the copyright to your article

Submit your next manuscript at springeropen.com 